114] P. Beckmann and A. Spizzichino, The Scattering of Electromagnetic Waves from Rough Surfaces. New York: Macmillan, 1963.

[15] R. E. Collin, "Electromagnetic scattering from perfectly conducting rough surfaces (A new full wave method)," IEEE Trans. Antennas Propagat., vol. 40, no. 12, pp. 1466-1477, 1992.

[16] J. C. Daley, W. J. Davis, and N. R. Mills, "Radar sea retums on high sea states," Naval Res. Lab., Washington, DC, Rep. 7142, U.S. Government Printing Office, 1970.

[17] M. E. Knotts. T. R. Michel, and K. A. O'Donnell, "Comparison of theory and experiment in light scattering from a randomly rough surface," $J$. Opt. Soc. Amer. A, vol. 10, no. 5, pp. 928-941, 1993.

18] J. S. Meditch, Stochastic Optimal Linear Estimation and Control. New York, 1989

119] S. O. Rice, "Reflection of electromagnetic waves from a slightly rough surface," Comm. Pure Appl. Math., vol. 4, pp. 251-378, 1951.

[20] M. I. Sancer, "Shadow corrected electromagnetic scattering from randomly rough surface," IEEE Trans. Antennas Propagat., vol. AP-17, pp. $577-585,1969$.

[21] J. A. Sanchez-Gil and M. Nieto-Vesperinas, "Light scattering from random rough dielectric surfaces," J. Opt. Soc. Amer. A, vol. 8, no. 8, pp. 1270-1286, 1991.

122] A. J. Sant, J. C. Dainty, and M. J. Kim, "Comparison of surface scattering between identical, randomly rough metal and dielectric diffusers," Opt. Lett., vol. 14, no. 21, pp. 1183-1186, 1989.

123] E. I. Thorsos and A. Ishimaru, "An examination of the full-wave method for rough surface scattering," paper presented at National Radio Science Meeting, Boulder, CO, 1988.

\section{Synthesis of Absorbing Boundary Conditions for the FDTD Method: Numerical Results}

\author{
E. N. M. Tromp and J. C. Olivier
}

Abstract-The communication presents numerical results in the form of a comparison of predictions due to a numerical algorithm using a previously published exact nonlocal Absorbing Boundary Condition (ABC) and analytical results for the field radiated by an infinitely small dipole. The numerical approximations are derived, and application to the FDTD method is straightforward. The method is compared to a related approach due to De Moerlose et al, and it is shown that the method yields better accuracy since higher order interpolation and numerical integration schemes may be used with this formulation.

\section{INTRODUCTION}

We consider a fundamental problem in the application of the Finite Difference Time Domain (FDTD) method [1] namely that of constructing suitable Absorbing Boundary Conditions (ABC's). The $\mathrm{ABC}$ truncates the volumetric computational domain electrically close to the scatterer of interest, but effectively simulates the extension of the computational domain to infinity. The accuracy of the ABC is a fundamental limitation on the accuracy of the FDTD method since any artificial reflections off the boundaries interfere with the fields in the computational domain. A number of articles dealing with ABC's have appeared in the literature [2]-[10]. Three types of ABC's, the mode annihilating, one-way wave equation approximations and nonlocal types of ABC's may be identified. Both the former type of $A B C$ 's can only absorb waves within a certain range of incident

Manuscript received December 27, 1993; revised July 11, 1994

E. N. M. Tromp is with the Twente University of Technology, Enschede The Netherlands.

J. C. Olivier is with the Department of Electrical and Electronic Engineering, University of Pretoria, Pretoria, South Africa.

IEEE Log Number 9408255.

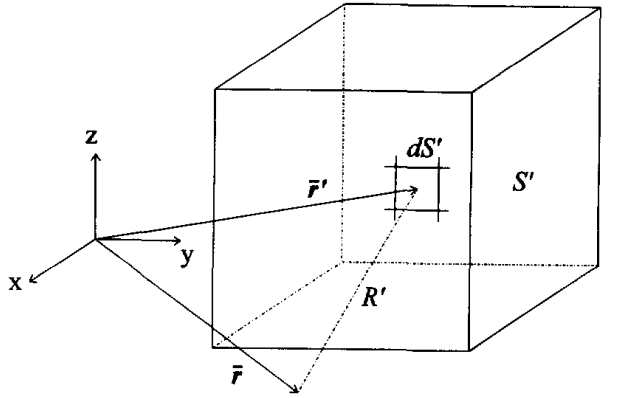

Fig. 1. The integration surface. $r /$ is the vector to the integration elemen $d S^{\prime}$ and $r$ is the observation point.. $R^{\prime}$ is the Euclidian distance between $\boldsymbol{r} /$ and $\boldsymbol{r}$

angles relative to the $\mathrm{ABC}$ plane and require a minimum spacing between the radiator/scatterers and the truncation planes. The third type of $\mathrm{ABC}$ is analytically exact and can be placed very close to the radiator/scatterer, but it requires the evaluation of a surface integral [11] for each point where the electromagnetic field is required. Potential benefits of the nonlocal $\mathrm{ABC}$ is drastic savings in computer memory, but at the cost of CPU time.

This communication presents numerical results for the analytical nonlocal $\mathrm{ABC}$ proposed in [8], and compares the results with those in [9] where a similar (related) nonlocal ABC based on the Kirchhoff formula was proposed. We will show that our method may be implemented with any higher order interpolation and numerical integration method and hence yields better accuracy, while the method of [9] is first order accurate with respect to the grid spacing and does not allow for higher order accuracy to be realized.

The communication is organized as follows. Section II will introduce the $\mathrm{ABC}$ and derive the numerical implementation. In Section III we will give numerical results and compare them to analytical results and those presented in [9]. Section IV concludes the communication.

\section{THE EXACT ABSORBING BOUNDARY}

CONDITION AND THE NUMERICAL IMPLEMENTATION

The nonlocal analytically exact $\mathrm{ABC}$ we consider was first derived in [8] and is given by

$$
\begin{aligned}
\frac{\partial \boldsymbol{H}(\boldsymbol{r}, t)}{\partial t} & =\frac{\partial}{\partial t} \frac{1}{4 \pi} \nabla \times \int_{S^{\prime}} \frac{\boldsymbol{n} \times \boldsymbol{H}\left(\boldsymbol{r}^{\prime}, t-\frac{R^{\prime}}{c}\right)}{R^{\prime}} d S^{\prime} \\
& -\frac{1}{4 \pi \mu} \nabla \times \nabla \times \int_{S^{\prime}} \frac{\boldsymbol{n} \times \boldsymbol{E}\left(\boldsymbol{r}^{\prime}, t-\frac{R^{\prime}}{c}\right)}{R^{\prime}} d S^{\prime} .
\end{aligned}
$$

The spacial operators may be placed inside the integral since they operate with respect to the unprimed coordinates. The various symbols are defined in Fig. 1 and the reader is referred to [8] for a full discussion of the $\mathrm{ABC}$

In order to test the $\mathrm{ABC}$ given by (1) we select a problem also used in [9] namely that of a small dipole antenna in the origin of a Cartesian coordinate system. It has a length $h$ that is very small compared with the radiated wavelength. If the current on the dipole is given by $f^{\prime}(t)$ and the dipole is directed along the unit vector $e_{s}$, the exact magnetic field at a point $r$ in space and time $t$ can be expressed as follows:

$$
\boldsymbol{H}(\boldsymbol{r}, t)=\frac{h}{4 \pi R}\left(\frac{f^{/ /}\left(t-\frac{R}{c}\right)}{c}+\frac{f^{\prime}\left(t-\frac{R}{c}\right)}{r}\right)\left(\boldsymbol{e}_{\mathrm{s}} \times \boldsymbol{e}_{r}\right)
$$




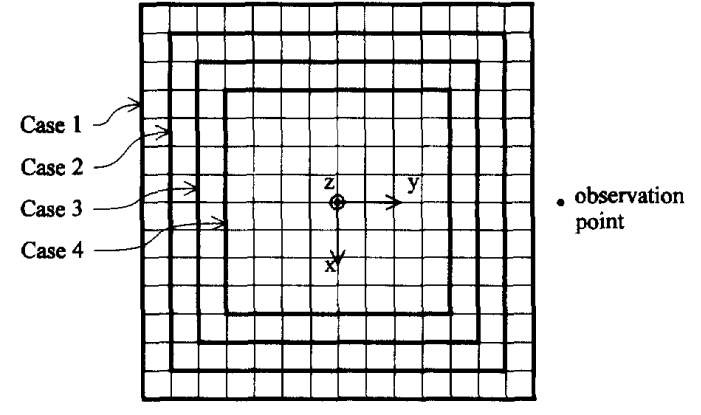

Fig. 2. Cross section $(z=0)$ of the Yee lattice and the integration surface in each of the four cases.

with

$$
R=\left|\boldsymbol{r}-\boldsymbol{r}_{s}\right| \boldsymbol{e}_{r}=\frac{\left|\boldsymbol{r}-\boldsymbol{r}_{s}\right|}{R}
$$

and in which $f^{\prime}$ represents the first derivative of $f$ with respect to $t$ and $f^{\prime /}$ the second. We integrate (1) with respect to time to get an exact expression for the $\boldsymbol{H}$-field as in (2)

$$
\begin{aligned}
\boldsymbol{H}(\boldsymbol{r}, t) & =\frac{1}{4 \pi} \nabla \times \int_{S^{\prime}} \frac{\boldsymbol{n} \times \boldsymbol{H}\left(\boldsymbol{r}^{\prime}, t-\frac{R^{\prime}}{c}\right)}{R^{\prime}} d S^{\prime} \\
& -\frac{1}{4 \pi \mu} \int_{-\infty}^{l} \nabla \times \nabla \times \int_{S^{\prime}} \frac{\boldsymbol{n} \times \boldsymbol{E}\left(\boldsymbol{r}^{\prime}, t-\frac{R^{\prime}}{c^{\prime}}\right)}{R^{\prime}} d S^{\prime} d t .
\end{aligned}
$$

We assume that the field components of the $\boldsymbol{E}$ and $\boldsymbol{H}$ fields are to be positioned on Yee grid cells. However, the relevant field components on the integration surface are not calculated with the FDTD method but the exact expressions (2) and (3) are used instead. In this way a clear picture of the accuracy of the $A B C$ can be obtained; in a real Yee lattice implementation some additional inaccuracy will be introduced by the FDTD method.

Both the surface integrals over the $\boldsymbol{H}$-field and over the $\boldsymbol{E}$-field in (4) must be done over the same surface. Since in a Yee lattice the tangential components of the $\boldsymbol{E}$-field in a certain plain are always at least a half cell width away from the same tangential components of the $\boldsymbol{H}$-field, it is necessary to interpolate at least one of the two field components to an accurate indication of both the field components in the same plane. This interpolation must be done perpendicular to the plane. If a fourth order Lagrange interpolation polynomial is used, the expression for the field just between the two nodes becomes simply:

$$
\begin{aligned}
\boldsymbol{H}(x, y, z)=\frac{1}{16} & (-\boldsymbol{H}(x-3 / 2 d x \cdot y \cdot \dot{y})+9 \boldsymbol{H}(x-1 / 2 d x, y, x) \\
& \left.+9 \boldsymbol{H}\left(x+\frac{1}{2} d x, y, z\right)-\boldsymbol{H}\left(x+\frac{3}{2} d x, y, \dot{z}\right)\right) \\
& +\boldsymbol{O}\left(d x^{5}\right)
\end{aligned}
$$

It is not necessary to store all four node quantities in time since the interpolation can be done after every time step in the FDTD method, so only one quantity for every node on the integration surface needs to be stored in time. Another problem is that the field needs to be retrieved at retarded time instances given by $r-R^{/} / c$. Since the fields are only known at certain discrete time instances, interpolation is once again used to find these values. The curl operators were approximated using central difference formulas.

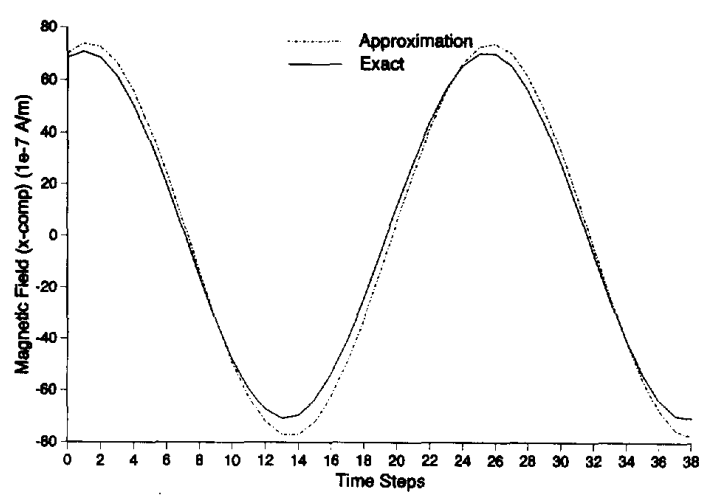

Fig. 3. Case 1: smallest distance between observation point and integration surface is one cell width.

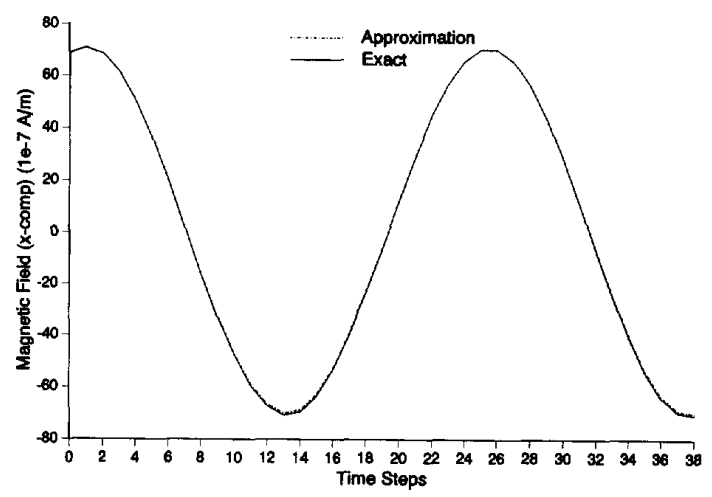

Fig. 4. Case 2: smallest distance between observation point and integration surface is two cell widths.

The surface integrations are done separately for each of the six boundary planes and then added. These are calculated using a second order Newton-Cotes formula.

\section{NUMERICAL Results}

The above described test case was implemented using a $14 \times 14 \times 14$ cell grid centered around the origin. The dipole was placed along the $z$-axis in the origin. The observation point was placed on the $y$-axis, one Yee cell width from the outside boundary. The $\boldsymbol{H}$-field on that point was evaluated numerically and compared to the exact value. This was done for four surfaces as indicated in Fig. 2. The cell dimensions were chosen as one tenth of the wavelength of the radiated field, and the time step was chosen according to the Courant condition as given in [12]. For a $10 \mathrm{MHz}$ source this implies a cell width of $3 \mathrm{~m}$ and a time step of $4.08 \mathrm{~ns}$. The results for case 1 and case 2 are shown in Figs. 3 and 4, respectively. For cases 2, 3, 4 the error is plotted in Fig. 5.

In order to form some form of comparison with other methods, we compare our case 2 to the results of [9] where a similar problem was tackled. The error reported in [9] was $2.3 \%$ and in our case it is $1.3 \%$. We can therefore conclude that our approach offers increased accuracy.

In [9] the nonlocal $\mathrm{ABC}$ was compared to the local Mur type $\mathrm{ABC}$ [4]. For the problem of case 2 above, the error of the nonlocal $A B C$ was significantly less than the error of the Mur $A B C$, but the latter $\mathrm{ABC}$ required orders of magnitude less $\mathrm{CPU}$ time. However, when the 


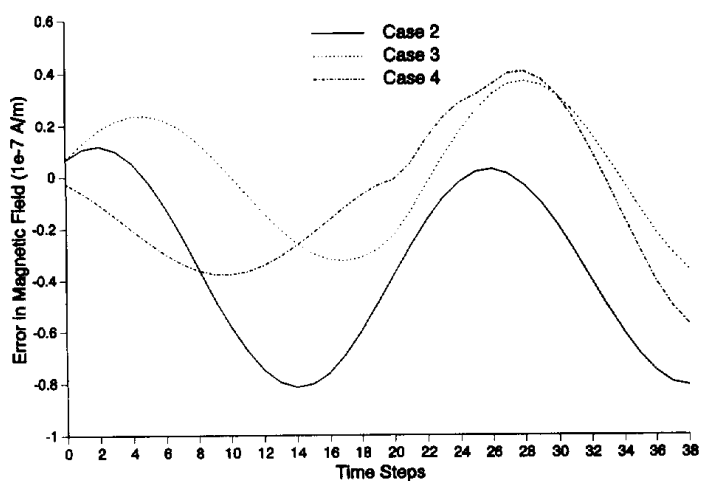

Fig. 5. The differences between the exact value of the $x$-component of the $\boldsymbol{H}$-field and the numerically approximated.

Mur $\mathrm{ABC}$ is placed far away from the source so that it yields the same accuracy as does the nonlocal $\mathrm{ABC}$, it required approximately a third of the CPU time. In this latter case, however, the computer memory requirement of the Mur $\mathrm{ABC}$ was orders of magnitude more than the requirements of the nonlocal $\mathrm{ABC}$. Hence, it is difficult to make general statements regarding which type of $\mathrm{ABC}$ is computationally more effective. In any event it is clear that research should be directed at finding ways of improving the $\mathrm{CPU}$ time required by the nonlocal $A B C$ 's if they are to be used instead of the usual local ABC's.

\section{CONCLUSIONS}

The exact nonlocal $A B C$ derived in [8] appears to be a useful and accurate tool to implement a nonreflecting ABC for the FDTD method. The accuracy is better than the nonlocal $A B C$ of [9] since this formulation allows higher order interpolation and integration which is not possible with the approach of [9]. When the accuracy of the current method and the local type of ABC's such as those due to Mur are required to be the same, the nonlocal $\mathrm{ABC}$ requires significantly less computer memory than does the Mur type ABC, but more CPU time than the Mur ABC.

Our method may be implemented with any order interpolation and integration schemes, so that we may increase the accuracy further if so desired with little computational overhead. This is in contrast to the method of [9] which is first order accurate. Research at reducing the CPU time requirements is continuing.

\section{REFERENCES}

[1] K. S. Yee, "Numerical solution of initial boundary value problems involving Maxwell's equations in isotropic media," IEEE Trans. Antennas Propagat., vol AP-14, no. 3, pp. 302-307, May 1966.

[2] A. Bayless and E. Turkel, "Radiation boundary conditions for wave-like equations," Pure Applied Math., vol. XXXIII, pp. 707-725, 1980.

[3] B. Engquist and A. Madja, "Absorbing boundary conditions for the numerical simulation of waves," Math. of Computation, vol. 31, no. 139, pp. 629-651, July 1977.

[4] G. Mur, "Absorbing boundary conditions for the finite difference approximation of the time-domain electromagnetic field equations," IEEE Trans. Electromag. Compat., vol. EMC-23, no. 4, pp. 377-382, Nov. 1981

[5] B. Gustafsson and H. O. Kreiss, "Boundary conditions for time dependant problems with an artificial boundary," J. Comput. Phys., vol. 30 , pp. 333-351, 1979.

[6] T. G. Moore, J. G. Blashak, A. Tavlove, and G. A. Kriegsman, "Theory and applications of radiation boundary operators," IEEE Trans. Antennas Propagat., vol AP-36, no. 12, pp. 1797-1811, Dec. 1988.
[7] J. B. Keller and D. Govoli, "Exact non-reflecting boundary conditions," J. Computat. Phys., vol. 82, pp. 172-192, 1989.

[8] J. C. Olivier, "On the synthesis of exact free space absorbing boundary conditions for the finite difference time domain method," IEEE Trans. Antennas Propagat., vol. 40, no. 4, pp. 456-460, Apr. 1992.

[9] J. De Moerlose and D. de Zutter, "Surface integral representation radiation boundary conditions for the FDTD method," IEEE Trans. Antennas Propagat., vol. 41, no. 7, pp. 890-896, July 1993.

[10] L. Ting and M. J. Miksis, "Exact boundary conditions for scattering problems," J. Acoust. Soc. Amer., vol. 80, pp. 1825-1827, 1986.

[11] O. M. Ramai and R. Mittra, "Finite element solution for a class of unbounded geometries," IEEE Trans. Antennas Propagat., vol. 39, no. 2, Feb. 1991 .

[12] A. Tavlove and M. E. Brown, "Numerical solution of steady-state electromagnetic scattering problems using the time-dependant Maxwell's equations," IEEE Trans. Microwave Theory Tech., vol. MTT-23, no. 8 , pp. 623-630, Aug. 1975.

\section{Superspheroids: A New Family of Radome Shapes}

\author{
P. L. Overfelt
}

Abstract - In the following we use the arc described by the twodimensional superquadric equation (taking its exponent $\nu$ to be any positive real number) in the first quadrant only and revolve it about its major axis to obtain a body of revolution family of geometric shapes called superspheroids. For certain values of length and radius and assuming that $1<\nu<2$, we have determined new shapes that are appropriate for high speed missile radomes. We have found that the superspheroid with optimized exponent value $\nu=1.381$ can almost exactly reproduce the traditional Von Karman radome geometry. Incidence angle maps and geometric properties have been determined for this superspheroidal family. We have used a ray tracing analysis to obtain boresight error induced by this family of shapes as a function of gimbal angle. The superspheroids are mathematically simple, can approximate most of the traditional radome geometries quite well, and are exceptionally easy to either program or use analytically.

\section{INTRODUCTION}

The two-dimensional superquadric equation [1]

$$
(x / a)^{\nu}+(y / b)^{\nu}=1
$$

has been applied recently to electromagnetic problems in the context of scattering from perfectly conducting superquadric cylinders [2] and also in the analysis of reflector antennas with superquadric aperture boundaries [3]. Generally $x$ and $y$ in (1) are assumed to run over the intervals $-a \leq x \leq a,-b \leq y \leq b$. In the following we write (1) in the form

$$
y=(b / a)\left(a^{\nu}-x^{\nu}\right)^{1 / \nu},
$$

$b \leq a, \nu$ any positive real number, and we keep in mind that in taking the $\nu$ th root, we are interested in real roots only. In (2) we allow $x$ to take on positive values only, i.e., $0 \leq x \leq a$. In this case (2) is an arc in the $x y$ plane which is completely above the $x$-axis except at the end point, $x=a$. By taking this arc, $y=f(x) \geq 0$, and revolving it about the $x$-axis, we obtain a body of revolution (BOR) given by

$$
y^{2}+z^{2}=(b / a)^{2}\left(a^{\nu}-x^{\nu}\right)^{2 / \nu} \text {, }
$$

Manuscript received March 7, 1994; revised September 16, 1994

The author is with the Physics Division, Research Department, Naval Air Warfare Center Weapons Division, China Lake, CA 93555-6001 USA. IEEE Log Number 9408254. 